\title{
The Relationship Among Integrin Alpha 7, CD133 and Nestin as Well as Their Correlation With Clinicopathological Features and Prognosis in Astrocytoma Patients
}

Changtai Li ( $\sim$ cai48527922@163.com )

Hospital of Chengdu Office of People's Government of Tibetan Autonomous Region https://orcid.org/0000-0002-9372-9452

Jiao Liu

Hospital of Chengdu Office of People's Government of Tibetan Autonomous Region

Wei Yang

Hospital of Chengdu Office of People's Government of Tibetan Autonomous Region

Chongyi Chen

Hospital of Chengdu Office of People's Government of Tibetan Autonomous Region

Bo Wu

Hospital of Chengdu Office of People's Government of Tibetan Autonomous Region

\section{Research}

Keywords: Integrin alpha-7, cancer stem cell markers, astrocytoma, WHO grade, prognosis

Posted Date: September 3rd, 2021

DOl: https://doi.org/10.21203/rs.3.rs-839387/v1

License: (c) (i) This work is licensed under a Creative Commons Attribution 4.0 International License.

Read Full License

Version of Record: A version of this preprint was published at Clinical Neurology and Neurosurgery on March 1st, 2022. See the published version at https://doi.org/10.1016/j.clineuro.2022.107198. 


\section{Abstract}

Objective: Integrin alpha 7 (ITGA7), a potential glioma stem cell marker, regulates several other stem cell markers including CD133 and Nestin in several cancers, meanwhile its high expression is related to poor prognosis in multiple solid tumor patients. However, few studies report correlation of ITGA7 with prognosis in astrocytoma patients. Hence, this study aimed to determine the astrocytoma-tissue ITGA7, CD133 and Nestin expressions to explore their relationship and clinical value for astrocytoma management.

Methods: Totally, 124 patients with primary astrocytoma were included. Their tumor tissue ITGA7, CD133 and Nestin expressions were determined by immunohistochemical $(\mathrm{IHC})$ assay and scored by intensity and density ranging from 0-12 points. Besides, their clinical features (such as world health organization (WHO) grade, isocitrate dehydrogenase (IDH) mutation, and adjuvant therapy etc.) were collected, also their overall survival (OS) were analyzed by follow-up data.

Results: The mean IHC scores for ITGA7, CD133 and Nestin were 4.9 $\pm 2.5,2.1 \pm 2.6$ and 5.8 \pm 2.6 , respectively. Moreover, ITGA7 high expression correlated with absence of IDH mutation $(P=0.004)$, advanced WHO grade $(P=0.001)$ and shorter OS $(P=0.005)$. Besides, ITGA7 positively correlated with CD133 $(P=0.001)$ and Nestin $(P=0.001)$ expressions. Regarding CD133 and Nestin, their high expression also correlated with increased WHO grade and shorter OS. Furthermore, multivariant Cox's regression analysis displayed that only CD133 high expression $(P=0.021)$ could independently predict reduced OS, while ITGA7 or Nestin high expression could not.

Conclusion: ITGA7, CD133 and Nestin are intercorrelated, also their high expressions associate with deteriorating disease conditions and poor prognosis in astrocytoma patients.

\section{Introduction}

Astrocytoma, as the most frequent central nervous system (CNS) tumor, has a high incidence in males compared to females with a ratio of 1.4:1 [1-3]. Regarding its management, different options are applied according to the world health organization (WHO) classification of astrocytoma [1]. In detail, surgery following radiotherapy is commonly applied in low grade astrocytoma patients (WHO grade I and grade II), while surgery in combination with adjuvant chemotherapy and/or radiotherapy is used to treat high grade astrocytoma patients (WHO grade III and WHO grade IV) since most astrocytomas in this classification are in diffuse form and may cause intracranial metastasis [4]. However, even after appropriate management, some astrocytoma patients (such as anaplastic astrocytoma patients) experience multiple, rapid recurrence which further decreases their long-term survival profile $[2,5,6]$. Therefore, it is necessary and urgent to discover novel biomarkers to improve prognosis in astrocytoma patients.

Integrins are large, membrane-spanning, heterodimeric cell-surface proteins that contribute to cell proliferation, differentiation, adhesion and migration by interacting with extracellular matrix (ECM) and 
cytoskeleton [7,8]. Among which, integrin alpha-7 (ITGA7) is a key mediator in rectal cancer, breast cancer and astrocytoma pathogenesis [9-12]. In addition, in the clinical field, ITGA7 high expression correlates with poor prognosis in clear renal cell carcinoma patients, rectal cancer patients and breast cancer patients $[10,13,14]$. Furthermore, ITGA7 not only acts as a potential cancer stem cell (CSC) marker, but also regulates multiple other CSC marker expressions (including CD44, CD90, CD133, SOX2, OCT3/4 and NANOG), meanwhile, these CSC markers (such as CD133 and Nestin) correlate with shorter overall survival (OS) in astrocytoma patients [9,11,15-17]. Given the fact that ITGA7 regulates CSC marker expressions in several cancers, besides CD133 and Nestin are well-characterised CSC markers in astrocytoma, we hypothesized that ITGA7 assessment might have a potentially clinical value in astrocytoma patients [18-21]. However, no relevant research has been conducted yet. Herein, we performed this study and aimed to explore the relationship among ITGA7, CD133 and Nestin, as well as to explore their correlation with clinicopathological features and prognosis in astrocytoma patients.

\section{Materials And Methods}

\subsection{Patients and specimens}

The retrospective study was carried out with the approval from the Institutional Review Board of Hospital of Chengdu Office of People's Government of Tibetan Autonomous Region (Hospital. C.T). Totally, 124 patients with primary astrocytoma who underwent surgical resection in the hospital between January 2016 and June 2020 were included in this study. By reviewing their clinical data, only patients who met the following criteria were screened out for study analysis: (i) histologically-confirmed astrocytoma in accord with World Health Organization (WHO) classification criteria [22]; (ii) age $\geq 18$ years; (iii) directly treated by surgical resection without neoadjuvant therapy; (iv) formalin fixed and paraffin embedded tissue (FFEPT) of astrocytoma was well preserved and available for immunohistochemical (IHC) assessment; (v) major clinical features, treatment information, and survival data were integral and available; (vi) no history of other malignant diseases; (vii) without history of exposure to radiotherapy or chemotherapy before diagnosis of astrocytoma. The written informed consents were obtained from the patients or their relatives. A total of 124 astrocytoma FFEPT specimens were acquired form the hospital's sample library, which were used for determination of ITGA7, CD133 and Nestin expression by IHC assay.

\subsection{Data compilation}

The major clinical feature data and treatment information, including patients' age, gender, WHO grade (2016 WHO Classification [22]), isocitrate dehydrogenase (IDH) mutation, preoperative Karnofsky Performance Scale (KPS) score and adjuvant therapy, were collected from the medical documents. In addition, follow-up records of patients were reviewed to abstract the survival information for estimation of overall survival (OS).

\subsection{IHC assay}


The FFEPT specimens were sliced into sections at a thickness of $4 \mu \mathrm{m}$, which were then deparaffinized with xylene, rehydrated with ethanol, and treated with hydrogen peroxide, followed by blocking with normal goat serum. Afterwards, the sections were respectively incubated overnight with primary antibodies (Invitrogen, Carlsbad, California, USA), including ITGA7 Polyclonal Antibody (1:25 dilutions), CD133 Polyclonal Antibody (1:500 dilutions) and Nestin Polyclonal Antibody (1:1000 dilutions).

Following that, the sections were incubated with Goat anti-Rabbit IgG $(H+L)$ Secondary Antibody (1:5000 dilutions, Invitrogen, Carlsbad, California, USA). Chromogenic reaction was realized using diaminobenzidine, followed by counterstaining with hematoxylin. At the end of $\mathrm{IHC}$ assay, the staining results of sections were viewed and photographed using a microscope. The intensity and density of the IHC staining were scored by two experts, and the detailed scoring procedures were performed in terms of the method described in the published literature $[23,9,24]$. The IHC score was the product of intensity score and density score, ranging from 0 to 12 . The average IHC score of the two experts was used as the final IHC score. In the analysis, the cut-off score of 3 was used to classify the high and low expression, that was: $\mathrm{IHC}$ score $\leq 3$, low expression; $\mathrm{IHC}$ score $>3$, high expression.

\subsection{Statistical analysis}

SPSS 26.0 (IBM Corp., Armonk, New York, USA) was applied for data analysis. GraphPad Prism 7.02 (GraphPad Software Inc., San Diego, California, USA) was used for graph construction. Correlation among three markers was checked using McNemar's test. Correlation of three markers with clinical features was analyzed by Chi-square test or Mantel-Haenszel Chi-square test. Correlation of three markers with OS was examined by Kaplan-Meier method and Log-rank test. Prognostic value of three markers was estimated using Cox's proportional hazard regression model analysis with forward stepwise method. A $P$ value $<0.05$ indicated a statistical significance.

\section{Results}

\subsection{Patients 'clinical characteristics}

The mean age of astrocytoma patients was $47.9 \pm 14.6$ years (Table 1). Besides, there were $77(62.1 \%)$ males and 47 (37.9\%) females in astrocytoma patients. Regarding their WHO grade, 5 (4.0\%), 53 (42.8\%), $45(36.3 \%)$ and $21(16.9 \%)$ patients were diagnosed as WHO grade I, WHO grade II, WHO grade III and WHO grade IV astrocytomas, respectively. Furthermore, 64 (51.6\%) cases presented with IDH mutation. The detailed clinical features were listed in Table 1.

\subsection{ITGA7, CD133 and Nestin expressions}

In astrocytoma patients, the mean IHC scores for ITGA7, CD133 and Nestin were $4.9 \pm 2.5,2.1 \pm 2.6$ and 5.8 \pm 2.6 , respectively (Figure 1A-D). For further analysis, ITGA7, CD133 and Nestin expressions were categorised into high or low expression based on a cut-off value of 3 on IHC score. Besides, there were 79 (63.7\%) cases presented with ITGA7 high expression, while 45 (36.3\%) cases presented with ITGA7 low expression. Meanwhile, there were 32 (25.8\%) cases presented with CD133 high expression, while 92 
(72.4\%) cases presented with CD133 low expression. Also, there were 91 (73.4\%) cases presented with Nestin high expression, while 33 (26.6\%) cases presented with Nestin low expression.

Regarding their relationship, ITGA7 expression positively correlated with CD133 expression and Nestin expression (both $P=0.001$ ) in astrocytoma patients (Table 2). Moreover, CD133 expression positively associated with Nestin expression in astrocytoma patients $(P=0.002)$.

\subsection{Correlation of ITGA7 expression with clinical features}

In astrocytoma patients, ITGA7 high expression was correlated with elevated WHO grade $(P=0.001)$ (Figure 2C) and absence of IDH mutation ( $P=0.004)$ (Figure 2D). While there was no correlation of ITGA7 expression with age ( $P=0.265)$ (Figure 2A), gender $(P=0.257)$ (Figure 2B), KPS score $(P=0.696)$ (Figure 2E), adjuvant radiotherapy ( $P=0.079)$ (Figure $2 \mathrm{~F}$ ) or adjuvant chemotherapy $(P=0.545)$ (Figure $\mathbf{2} \mathbf{G}$ ).

\subsection{Correlation of CD133 and Nestin expressions with clinical features}

CD133 high expression positively correlated with WHO grade in astrocytoma patients $(P=0.012)$ (Figure 3C). However, there was no correlation of $C D 133$ expression with age $(P=0.728)$ (Figure 3A), gender $(P=0.186)$ (Figure 3B), IDH mutation ( $P=0.149)$ (Figure 3D), KPS score $(P=0.622)$ (Figure 3E), adjuvant radiotherapy $(P=0.121)$ (Figure $3 \mathbf{F})$ or adjuvant chemotherapy $(P=0.308)$ (Figure 3G).

Regarding Nestin, its high expression correlated with advanced WHO stage $(P<0.001)$ (Figure 3J), absence of IDH mutation ( $P=0.043$ ) (Figure $3 \mathrm{~K}$ ) and presence of adjuvant radiotherapy $(P=0.043)$ (Figure $3 \mathrm{M})$ in astrocytoma patients. While no correlation of Nestin expression with age $(P=0.481)$ (Figure $3 \mathrm{H})$, gender $(P=0.528)$ (Figure $3 \mathrm{I})$, KPS score $(P=0.334)$ (Figure $3 \mathrm{~L})$, or adjuvant chemotherapy $(P=0.877)$ (Figure $3 \mathrm{~N}$ ) was observed.

\subsection{Correlation of ITGA7, CD133 and Nestin expressions with OS}

ITGA7 high expression ( $P=0.005)$ (Figure 4A), CD133 high expression ( $P=0.006)$ (Figure 4B) and Nestin high expression $(P=0.003)$ (Figure $4 \mathrm{C})$ all correlated with reduced OS in astrocytoma patients.

Univariant Cox's regression analysis revealed that ITGA7 high expression $(P=0.007)$, CD133 high expression $(P=0.007)$ and Nestin high expression $(P=0.006)$ all correlated with reduced $O S$ in astrocytoma patients (Figure 5A). Further multivariant Cox's regression analysis displayed that only CD133 high expression ( $P=0.021)$ (Figure $5 \mathrm{~B}$ ) could independently predict reduced $0 \mathrm{~S}$ in astrocytoma patients, while ITGA7 high expression or Nestin high expression could not.

\section{Discussion}

ITGA7 is a cell adhesion molecule which is critical for cell migration, homeostasis and translocation $[7,8]$. Apart from its critical role in normal cell biology, ITGA7 is also able to induce cell malignant behaviours in several cancers $[8,7,9-11]$. One study displays that ITGA7 regulates phosphatidylinositol 3 kinase 
(PI3K)/protein kinase B (Akt) pathway to further promote glioma CSC proliferation and clonogenic survival [12]. Recently, ITGA7 has been identified as a novel biomarker in several cancer patients. For example, ITGA7 expression positively correlates with pathological grade and TNM stage in clear cell renal cell carcinoma patients, breast cancer patients and rectal cancer patients $[10,14,13]$. Furthermore, ITGA7 high expression is related to worse OS in rectal cancer patients and breast cancer patients $[10,14]$. However, limited study can be found in terms of the correlation of ITGA7 with prognosis in astrocytoma patients. Therefore, we performed this study and discovered that ITGA7 high expression correlated with advanced WHO grade and poor prognosis in astrocytoma patients. The possible reasons to explain these findings were: (a) ITGA7 might promote astrocytoma cell proliferation, invasion and metastasis through the regulation of $\mathrm{PI} 3 \mathrm{~K} / \mathrm{Akt}$ pathway, therefore led to deteriorating clinical features in astrocytoma patients $[12,17]$. (b) ITGA7 might regulate ERK signalling pathway to mediate cell differentiation, thereby led to increased WHO grade in astrocytoma patients [25]. (c) Regarding their survival profile, ITGA7 high expression might lead to increased chance of developing chemotherapy resistance due to the clonogenic survival of CSC, therefore resulted in poor prognosis in astrocytoma patients [26].

Great attention has been paid in discovering the properties of CSC since they are responsible for cancer metastasis, recurrence and drug resistance $[27,28]$. CD133 and Nestin are two heavily investigated CSC markers, meanwhile they are commonly co-expressed in neural stem cells [29]. In the clinical field, CD133 and Nestin correlated with advanced WHO grade, also their high expressions correlate with shorter OS in astrocytoma patients [15]. However, no relevant research reports their relationship with ITGA7 in astrocytoma patients. In the present study, we discovered that ITGA7 expression positively correlated with CD133 and Nestin expressions in astrocytoma patients. Besides, both CD133 and Nestin expressions correlated with advanced WHO stage and unfavourable survival profile in astrocytoma patients.

Furthermore, CD133 high expression could independently predict shorter OS in astrocytoma patients, which could be explained as follows: (a) Regarding the correlation of ITGA7 expression with CD133 and Nestin expressions, high ITGA7 expression suggested increased CSC cell proportion and density as discussed earlier, meanwhile CD133 and Nestin were glioma CSC markers, therefore, ITGA7 positively correlated with CD133 and Nestin in astrocytoma patients [29]. (b) In terms of the correlation of CD133 and Nestin expression with WHO grade, elevated CSC marker expressions (CD133 and Nestin in this case) suggested inhibition of glioma CSC apoptosis while promotion of CSC angiogenesis through Wnt/ $\beta$ catenin and PI3K/Akt pathways, therefore led to clonogenic survival of glioma CSC and further led to differentiation of glioma CSC into astrocytoma cells, which resulted in elevated astrocytoma cell density and irregular nuclear morphology, therefore led to increased WHO grade in astrocytoma patients $[27,28]$. (c) In terms of the correlation of CD133 and Nestin with prognosis, elevated CSC marker expressions (CD133 and Nestin) indicated a higher proportion of CSC, which led to increased chance of developing drug resistance and distal metastasis, therefore resulted unfavourable survival profile in astrocytoma patients $[28,27]$.

There were several limitations in the current study. Firstly, the interacting mechanisms among ITGA7, CD133 and Nestin was not discovered in the present study, and further study was needed. Secondly, the sample size in our study was relatively small, therefore further study with larger sample size to validate 
the prognostic value of these biomarkers was needed. Thirdly, we detected ITGA7, CD133 and Nestin expressions from astrocytoma tissue samples harvested from patients, therefore these marker expressions from blood samples were not detected and further study could investigate this aspect. Finally, further experiments were warranted to determine the effect of ITGA7 on chemotherapy resistance in astrocytoma patients.

In conclusion, ITGA7, CD133 and Nestin are intercorrelated, also their high expressions correlate with deteriorating disease condition and poor prognosis in astrocytoma patients, suggesting their potentially clinical value for astrocytoma management.

\section{Declarations}

\section{Ethics approval and consent to participate}

The retrospective study was carried out with the approval from the Institutional Review Board of Hospital of Chengdu Office of People's Government of Tibetan Autonomous Region (Hospital. C.T). The written informed consents were obtained from the patients or their relatives.

\section{Consent for publication}

Not applicable.

\section{Availability of data and material}

The datasets generated during and/or analyzed during the current study are available from the corresponding author on reasonable request.

\section{Competing interests}

The authors have no conflicts of interest to declare.

\section{Funding}

None.

\section{Authors' contributions}

$\mathrm{CL}$ and $\mathrm{JL}$ conceived, designed, performed all experiments and wrote the manuscript. WY and CC confirm the authenticity of all the raw data. BW was responsible for the collection and follow-up of clinical cases. WY and BW were responsible for data statistics. All authors have read and approved the manuscript.

\section{Acknowledgements}

None. 


\section{References}

1. Ostrom QT, Bauchet L, Davis FG, Deltour I, Fisher JL, Langer CE, Pekmezci M, Schwartzbaum JA, Turner MC, Walsh KM, Wrensch MR, Barnholtz-Sloan JS (2014) The epidemiology of glioma in adults: a "state of the science" review. Neuro Oncol 16 (7):896-913. doi:10.1093/neuonc/nou087

2. Chen R, Smith-Cohn M, Cohen AL, Colman H (2017) Glioma Subclassifications and Their Clinical Significance. Neurotherapeutics 14 (2):284-297. doi:10.1007/s13311-017-0519-x

3. Hirtz A, Rech F, Dubois-Pot-Schneider H, Dumond H (2020) Astrocytoma: A Hormone-Sensitive Tumor? Int J Mol Sci 21 (23). doi:10.3390/ijms21239114

4. Tom MC, Cahill DP, Buckner JC, Dietrich J, Parsons MW, Yu JS (2019) Management for Different Glioma Subtypes: Are All Low-Grade Gliomas Created Equal? Am Soc Clin Oncol Educ Book 39:133-145. doi:10.1200/EDBK_238353

5. Kulac I, Tihan T (2019) Pilomyxoid astrocytomas: a short review. Brain Tumor Pathol 36 (2):52-55. doi:10.1007/s10014-019-00343-0

6. Ellis JA, Waziri A, Balmaceda C, Canoll P, Bruce JN, Sisti MB (2009) Rapid recurrence and malignant transformation of pilocytic astrocytoma in adult patients. J Neurooncol 95 (3):377-382. doi:10.1007/s11060-009-9935-z

7. Li ZH, Zhou Y, Ding YX, Guo QL, Zhao L (2019) Roles of integrin in tumor development and the target inhibitors. Chin J Nat Med 17 (4):241-251. doi:10.1016/S1875-5364(19)30028-7

8. Campbell ID, Humphries MJ (2011) Integrin structure, activation, and interactions. Cold Spring Harb Perspect Biol 3 (3). doi:10.1101/cshperspect.a004994

9. Lv Z, Yang Y, Yang C (2020) Integrin alpha7 correlates with worse clinical features and prognosis, and its knockdown inhibits cell proliferation and stemness in tongue squamous cell carcinoma. Int J Oncol 56 (1):69-84. doi:10.3892/ijo.2019.4927

10. Bai X, Gao C, Zhang L, Yang S (2019) Integrin alpha7 high expression correlates with deteriorative tumor features and worse overall survival, and its knockdown inhibits cell proliferation and invasion but increases apoptosis in breast cancer. J Clin Lab Anal 33 (8):e22979. doi:10.1002/jcla.22979

11. Xia D, Chen B, Yang X (2019) Correlation of integrin alpha 7 with clinicopathological characteristics and survival profiles, as well as its regulatory role in cell proliferation, apoptosis, and stemness in nonsmall-cell lung cancer. J Clin Lab Anal 33 (8):e22973. doi:10.1002/jcla.22973

12. Haas TL, Sciuto MR, Brunetto L, Valvo C, Signore M, Fiori ME, di Martino S, Giannetti S, Morgante L, Boe A, Patrizii M, Warnken U, Schnolzer M, Ciolfi A, Di Stefano C, Biffoni M, Ricci-Vitiani L, Pallini R, De 
Maria R (2017) Integrin alpha7 Is a Functional Marker and Potential Therapeutic Target in Glioblastoma. Cell Stem Cell 21 (1):35-50 e39. doi:10.1016/j.stem.2017.04.009

13. Chen Y, Wang Y, Cai Z, Fan X, Zhang Y (2020) Integrin alpha7 is overexpressed and correlates with higher pathological grade, increased T stage, advanced TNM stage as well as worse survival in clear cell renal cell carcinoma patients: A retrospective study. J Clin Lab Anal 34 (1):e23034.

doi:10.1002/jcla.23034

14. Huang J, Tian Y, Chen J (2018) Overexpression of integrin alpha7 correlates with advanced disease condition and poor prognosis in rectal cancer patients. Int J Clin Exp Pathol 11 (9):4630-4636

15. Zhang Q, Xu B, Chen J, Chen F, Chen Z (2020) Clinical significance of CD133 and Nestin in astrocytic tumor: The correlation with pathological grade and survival. J Clin Lab Anal 34 (3):e23082. doi:10.1002/jcla.23082

16. Ming XY, Fu L, Zhang LY, Qin YR, Cao TT, Chan KW, Ma S, Xie D, Guan XY (2016) Integrin alpha7 is a functional cancer stem cell surface marker in oesophageal squamous cell carcinoma. Nat Commun 7:13568. doi:10.1038/ncomms13568

17. Ge JC, Wang YX, Chen ZB, Chen DF (2020) Integrin alpha 7 correlates with poor clinical outcomes, and it regulates cell proliferation, apoptosis and stemness via PTK2-PI3K-Akt signaling pathway in hepatocellular carcinoma. Cell Signal 66:109465. doi:10.1016/j.cellsig.2019.109465

18. Barzegar Behrooz A, Syahir A, Ahmad S (2019) CD133: beyond a cancer stem cell biomarker. J Drug Target 27 (3):257-269. doi:10.1080/1061186X.2018.1479756

19. Aghajani M, Mansoori B, Mohammadi A, Asadzadeh Z, Baradaran B (2019) New emerging roles of CD133 in cancer stem cell: Signaling pathway and miRNA regulation. J Cell Physiol 234 (12):2164221661. doi:10.1002/jcp.28824

20. Rabea AR, Elshahid AR, Elhakim MS, Hashish YH, Jafferany M, Elsaie ML (2020) Therapeutic implications of immunohistochemical expression pattern of the cancer stem cell marker (nestin) in basal and squamous cell carcinomas. Dermatol Ther 33 (6):e13921. doi:10.1111/dth.13921

21. Costa CD, Justo AA, Kobayashi PE, Story MM, Palmieri C, Laufer Amorim R, Fonseca-Alves CE (2019) Characterization of OCT3/4, Nestin, NANOG, CD44 and CD24 as stem cell markers in canine prostate cancer. Int J Biochem Cell Biol 108:21-28. doi:10.1016/j.biocel.2019.01.002

22. Louis DN, Perry A, Reifenberger G, von Deimling A, Figarella-Branger D, Cavenee WK, Ohgaki H, Wiestler OD, Kleihues P, Ellison DW (2016) The 2016 World Health Organization Classification of Tumors of the Central Nervous System: a summary. Acta Neuropathol 131 (6):803-820. doi:10.1007/s00401-016$1545-1$ 
23. Shen S, Yao Y (2021) A-kinase interacting protein 1 is sufficiently expressed and positively associates with WHO grade, meanwhile predicts unfavorable overall survival independently in glioma patients. Medicine (Baltimore) 100 (4):e20426. doi:10.1097/MD.0000000000020426

24. Abd El hafez A, El-Hadaad HA (2014) Immunohistochemical expression and prognostic relevance of Bmi-1, a stem cell factor, in epithelial ovarian cancer. Ann Diagn Pathol 18 (2):58-62.

doi:10.1016/j.anndiagpath.2013.11.004

25. McClure MJ, Ramey AN, Rashid M, Boyan BD, Schwartz Z (2019) Integrin-alpha7 signaling regulates connexin 43, M-cadherin, and myoblast fusion. Am J Physiol Cell Physiol 316 (6):C876-C887. doi:10.1152/ajpcell.00282.2018

26. Al Amri WS, Baxter DE, Hanby AM, Stead LF, Verghese ET, Thorne JL, Hughes TA (2020) Identification of candidate mediators of chemoresponse in breast cancer through therapy-driven selection of somatic variants. Breast Cancer Res Treat 183 (3):607-616. doi:10.1007/s10549-020-05836-7

27. Ma Q, Long W, Xing C, Chu J, Luo M, Wang HY, Liu Q, Wang RF (2018) Cancer Stem Cells and Immunosuppressive Microenvironment in Glioma. Front Immunol 9:2924.

doi:10.3389/fimmu.2018.02924

28. Zhu K, Xie V, Huang S (2020) Epigenetic regulation of cancer stem cell and tumorigenesis. Adv Cancer Res 148:1-26. doi:10.1016/bs.acr.2020.05.001

29. Ludwig K, Kornblum HI (2017) Molecular markers in glioma. J Neurooncol 134 (3):505-512. doi:10.1007/s11060-017-2379-y

\section{Tables}

Table 1. Clinical characteristics of astrocytoma patients 


\begin{tabular}{lc}
\hline Items & Astrocytoma patients $(\mathrm{N}=124)$ \\
\hline Age (years), mean \pm SD & $47.9 \pm 14.6$ \\
$<60$ years & $92(74.2)$ \\
\hline$\geq 60$ years & $32(25.8)$ \\
\hline Gender, No. (\%) & $77(62.1)$ \\
\hline Male & $47(37.9)$ \\
\hline Female & $5(4.0)$ \\
\hline WHO grade, No. (\%) & $53(42.8)$ \\
\hline I & $45(36.3)$ \\
\hline II & $21(16.9)$ \\
\hline III & $64(51.6)$ \\
\hline IV & $60.0(60.0-70.0)$ \\
\hline IDH mutation, No. (\%) & $69(55.6)$ \\
\hline KPS score, median (IQR) & $55(44.4)$ \\
\hline$<70$ & $101(81.5)$ \\
\hline$\geq 70$ & $84(67.7)$ \\
\hline Adjuvant treatment, No. (\%) & \\
\hline Radiotherapy & \\
\hline Chemotherapy & \\
\hline
\end{tabular}

SD, standard deviation; WHO, World Health Organization; IDH, isocitrate dehydrogenase;KPS, Karnofsky performance status; IQR, interquartile range.

Table 2. Correlation among ITGA7, CD133 and Nestin expressions

\begin{tabular}{lccccccc}
\hline Items & & \multicolumn{2}{c}{ ITGA7 } & P & \multicolumn{2}{c}{ Nestin } & P value \\
\cline { 3 - 4 } & & Low & High & value & Low & High & \\
& & $(\mathrm{n}=45)$ & $(\mathrm{n}=79)$ & & $(\mathrm{n}=33)$ & $(\mathrm{n}=91)$ & \\
\hline CD133 & Low $(\mathrm{n}=92)$ & $41(44.6)$ & $51(55.4)$ & 0.001 & $31(33.7)$ & $61(66.3)$ & 0.002 \\
& High $(\mathrm{n}=32)$ & $4(12.5)$ & $28(87.5)$ & $2(6.2)$ & $30(93.8)$ & \\
Nestin & Low $(\mathrm{n}=33)$ & $20(60.6)$ & $13(39.4)$ & 0.001 & - & - & - \\
& High $(\mathrm{n}=91)$ & $25(27.5)$ & $66(72.5)$ & & - & - & \\
\hline
\end{tabular}

ITGA7, integrin alpha7.

\section{Figures}


A

ITGA7 low expression (X200)

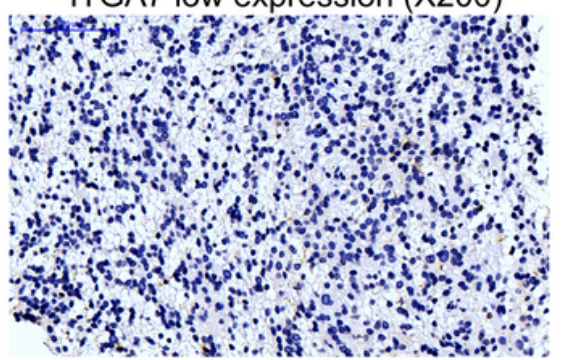

CD133 low expression (X200)

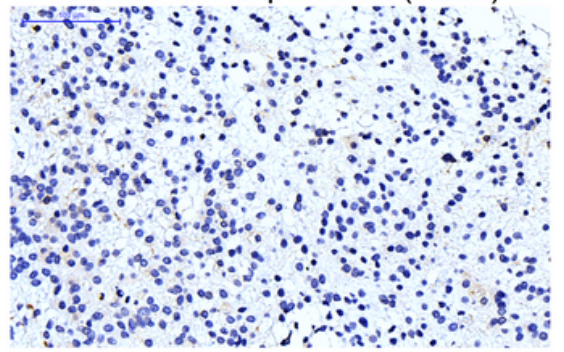

Nestin low expression (X200)

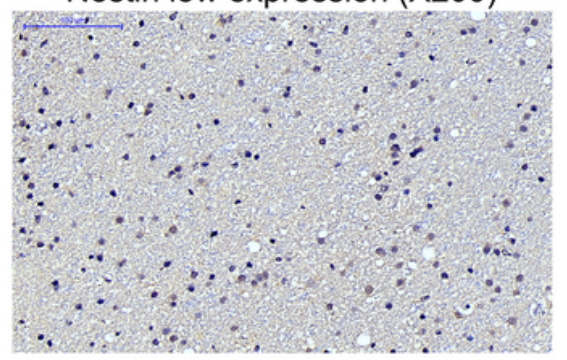

ITGA7 high expression (X200)

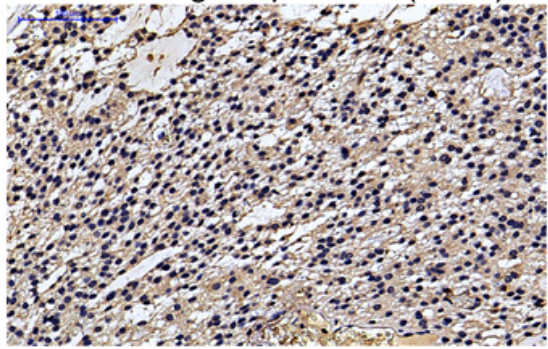

CD133 high expression (X200)

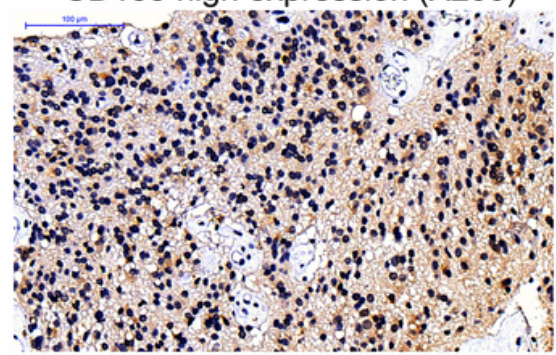

Nestin high expression (X200)

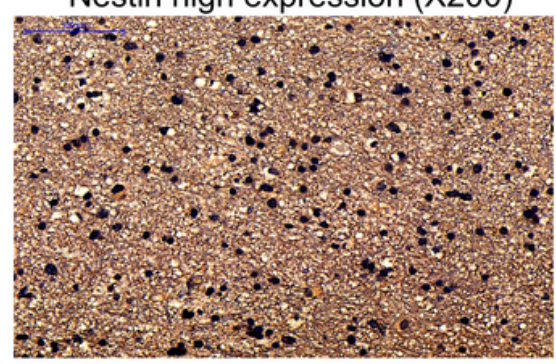

B

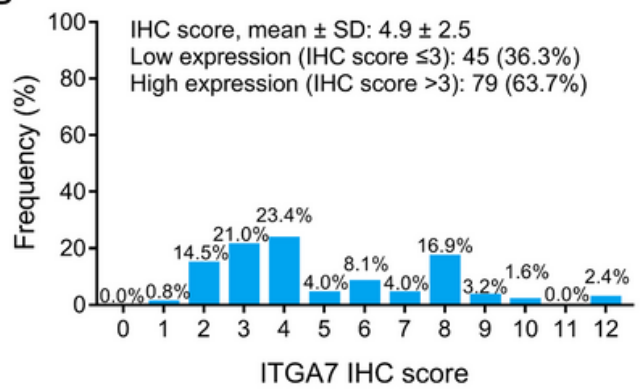

C

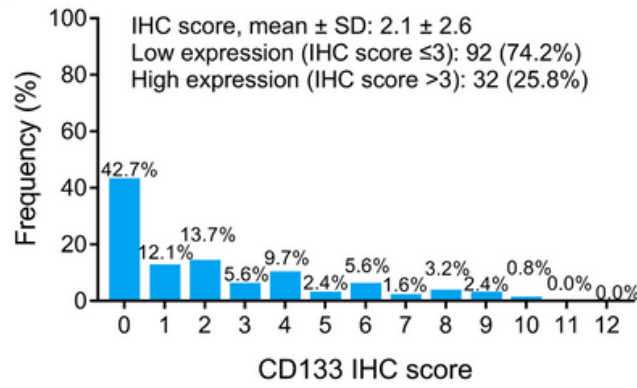

D

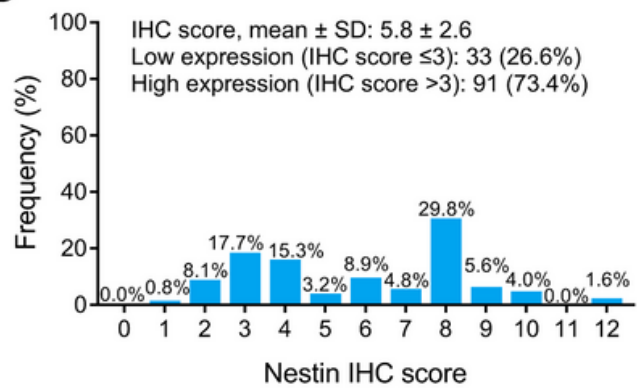

\section{Figure 1}

ITGA7, CD133 and Nestin IHC scores. The distribution of tumor tissue ITGA7 (A, B), CD133 (A, C) and Nestin (A, D) IHC scores in astrocytoma patients. ITGA7: Integrin alpha 7. IHC: immunohistochemical. 
A

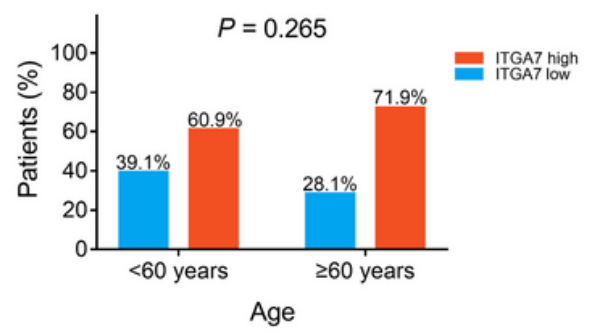

D

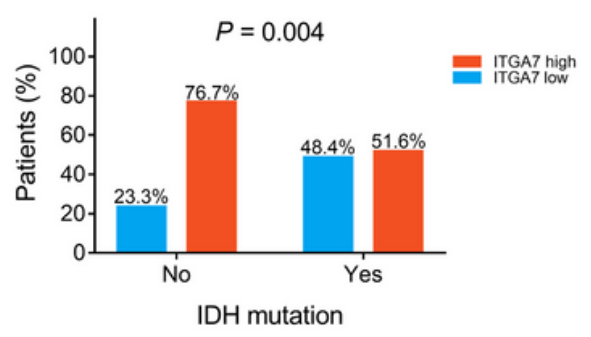

G

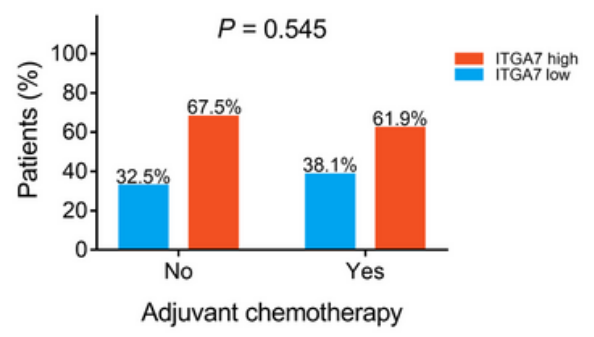

B

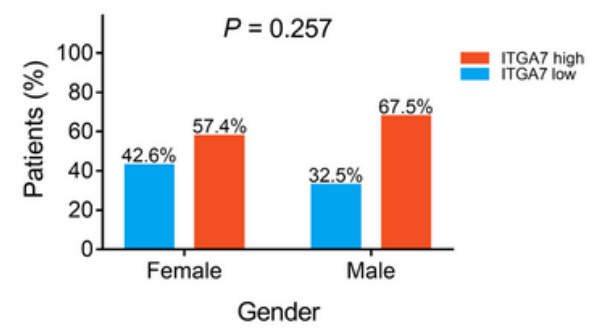

E

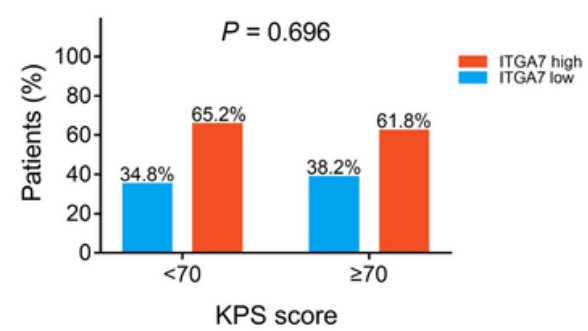

C

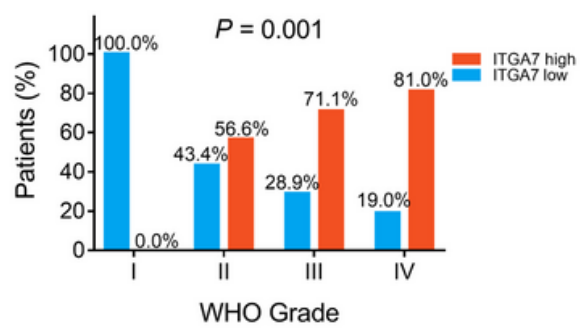

F

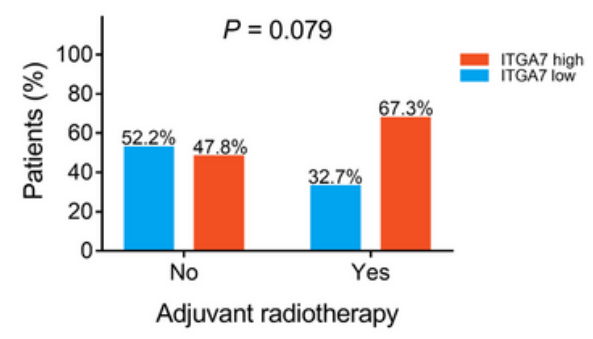

Figure 2

ITGA7 high expression correlated with absence of IDH mutation and elevated WHO grade. Correlation of ITGA7 expression with age (A), gender (B), WHO grade (C), IDH mutation (D), KPS score (E), adjuvant radiotherapy $(F)$ and adjuvant chemotherapy $(G)$ in astrocytoma patients. ITGA7: Integrin alpha 7, WHO: world health organisation, IDH: isocitrate dehydrogenase, KPS: Karnofsky Performance Scale. 
A

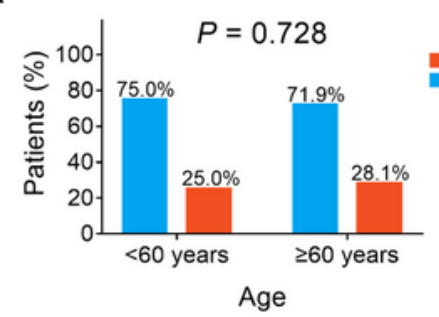

D

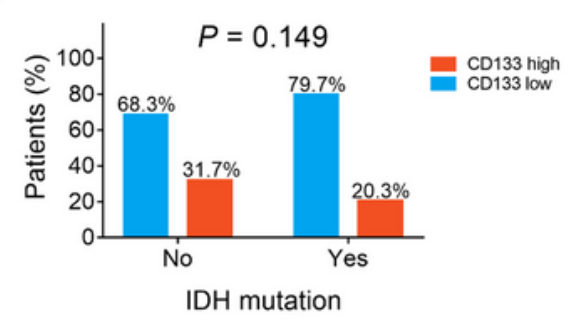

G

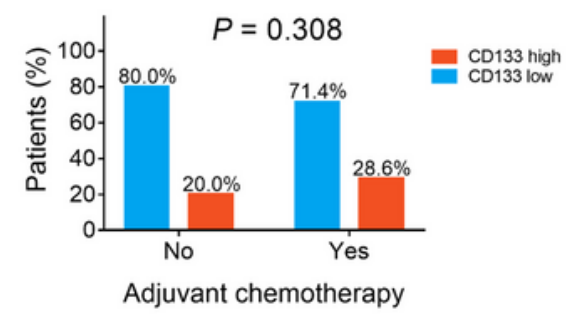

J

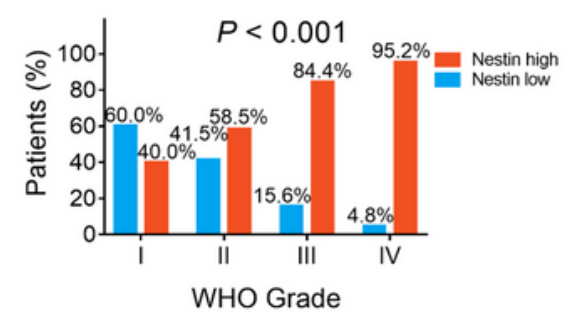

M

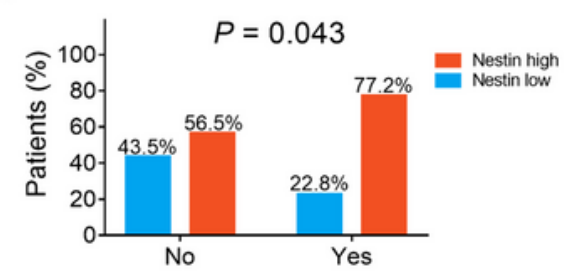

Adjuvant radiotherapy
B

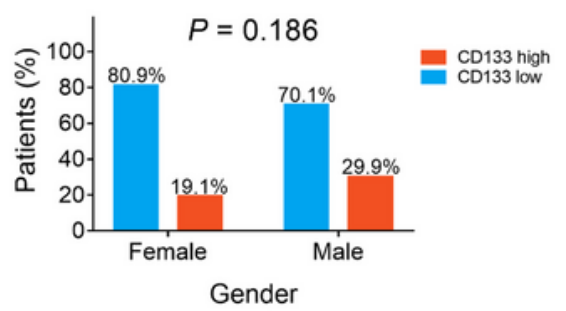

E

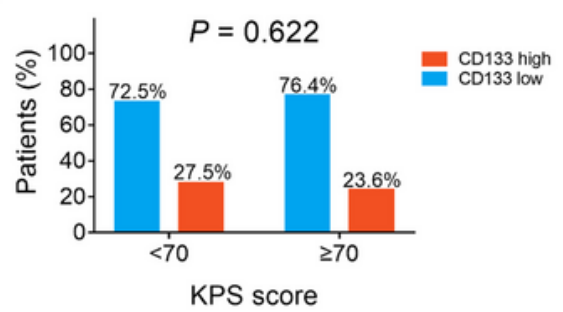

$\mathrm{H}$

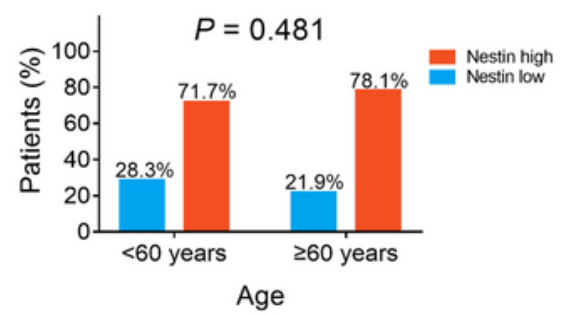

$\mathrm{K}$

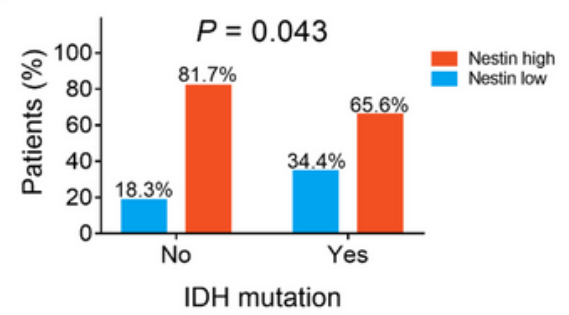

$\mathrm{N}$

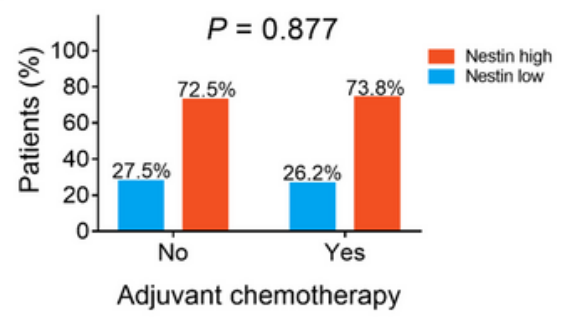

C

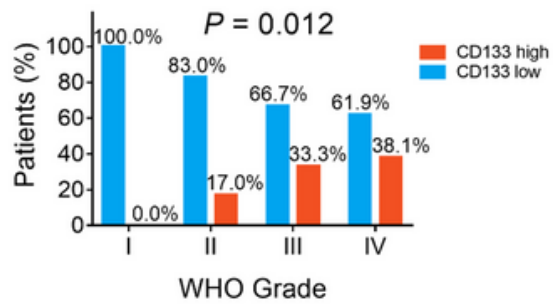

F

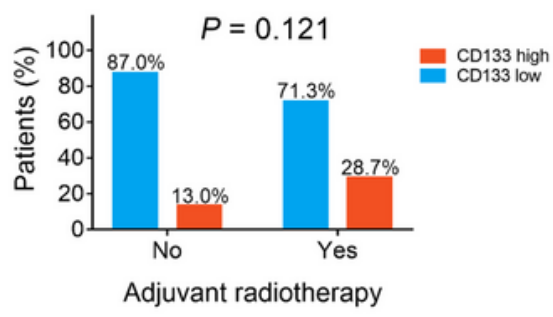

I

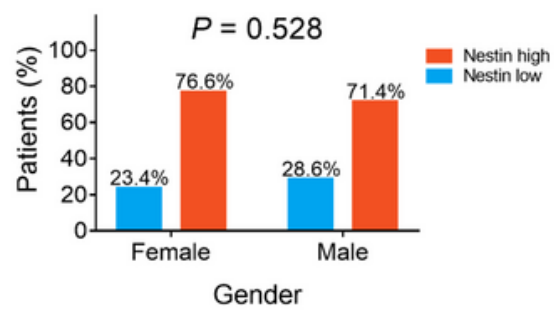

L

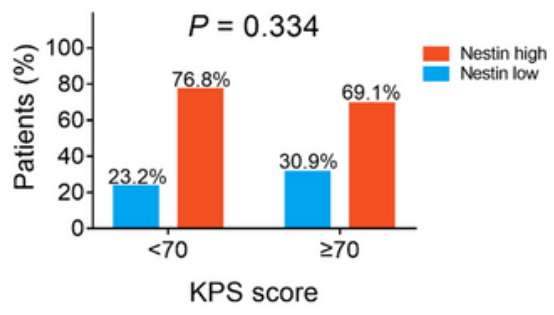

Figure 3

CD133 and Nestin high expressions correlated with increased WHO grade. Correlation of CD133 expression with age (A), gender (B), WHO grade (C), IDH mutation (D), KPS score (E), adjuvant radiotherapy $(F)$ and adjuvant chemotherapy $(G)$ in astrocytoma patients. Correlation of Nestin expression with age $(\mathrm{H})$, gender $(\mathrm{I})$, WHO grade $(\mathrm{J})$, IDH mutation $(\mathrm{K})$, KPS score $(\mathrm{L})$, adjuvant radiotherapy 
(M) and adjuvant chemotherapy $(\mathrm{N})$ in astrocytoma patients. WHO: world health organisation, IDH: isocitrate dehydrogenase, KPS: Karnofsky Performance Scale.

A

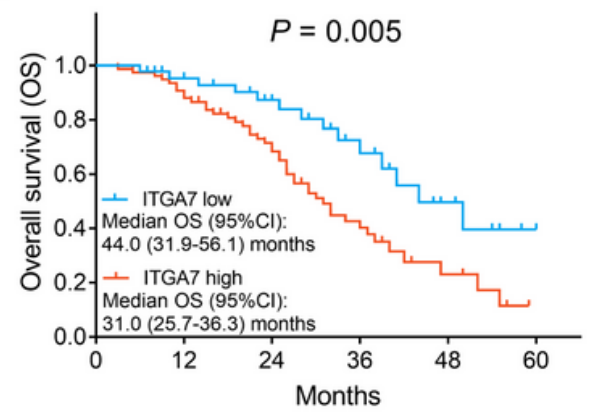

B

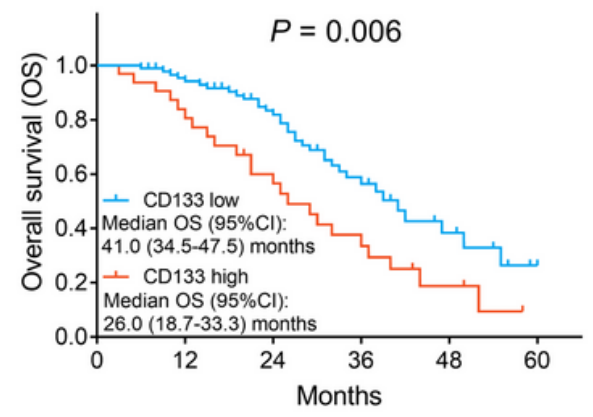

C

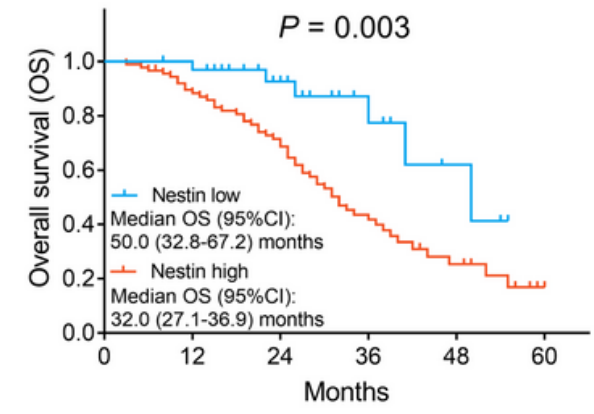

Figure 4

ITGA7, CD133 and Nestin high expressions correlated with shorter OS. ITGA7 (A), CD133 (B) and Nestin (C) high expression correlated with shorter OS in astrocytoma patients. ITGA7: Integrin alpha 7, OS: overall survival. 
A

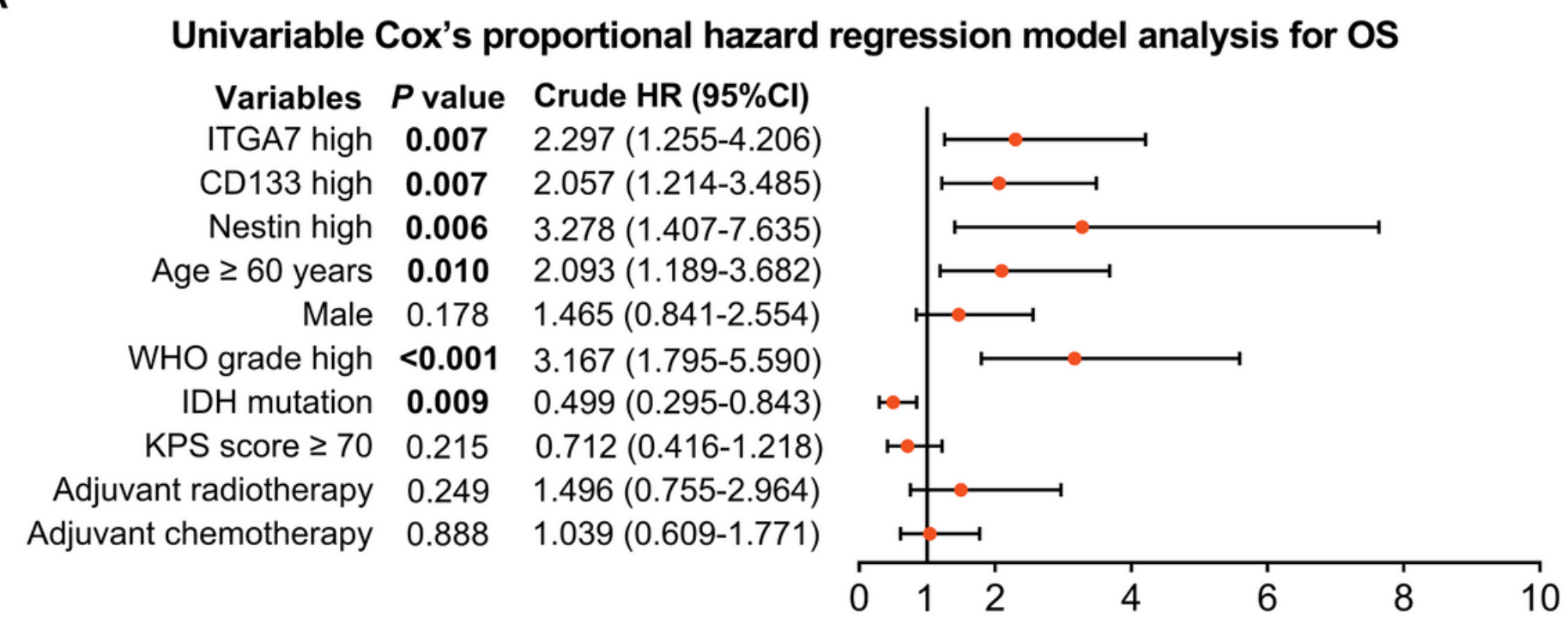

B

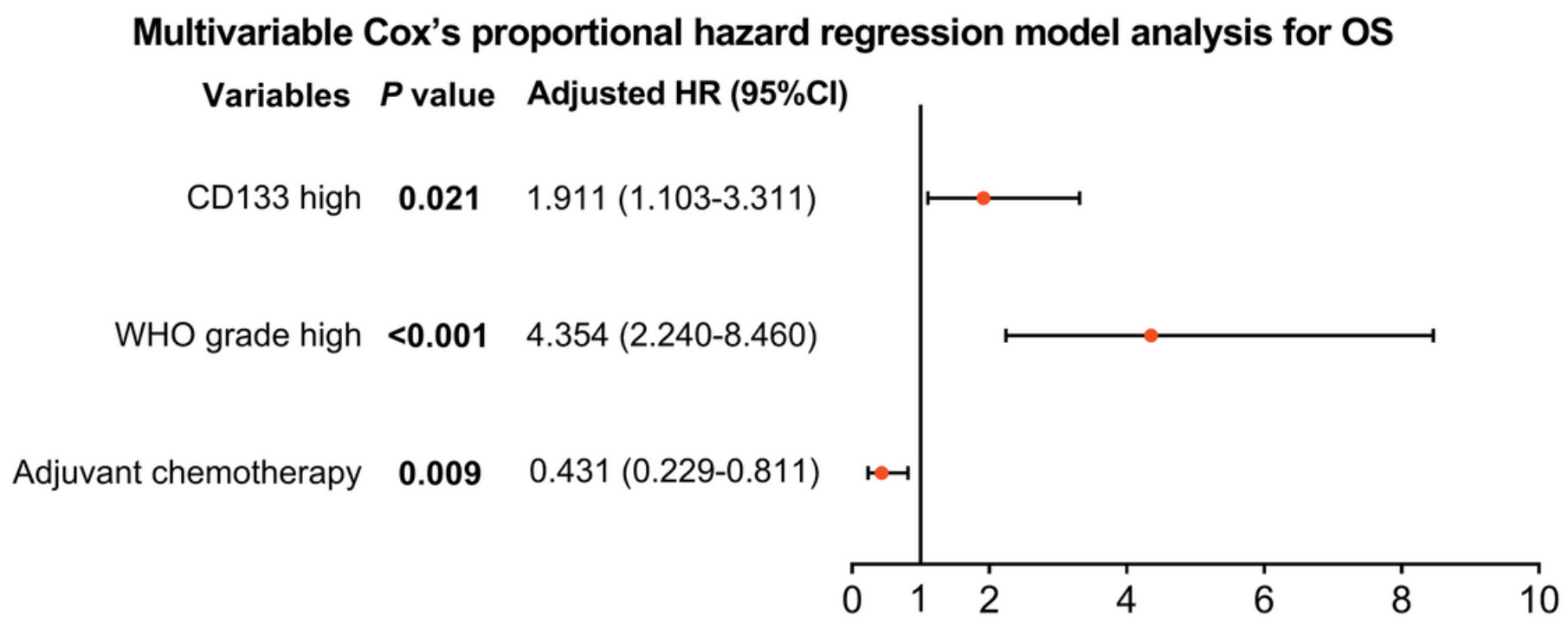

Figure 5

CD133 high expression independently correlated with reduced OS. Univariant (A) and multivariate (B) Cox's regression analyses of factors related to OS in astrocytoma patients. ITGA7: Integrin alpha 7, OS: overall survival, WHO: world health organisation, IDH: isocitrate dehydrogenase, KPS: Karnofsky Performance Scale. 